\title{
LA CONVENCIÓN DEL PATRIMONIO MUNDIAL EN UN MUNDO GLOBALIZADO
}

\section{THE WORLD HERITAGE CONVENTION IN A GLOBALIZED WORLD}

\author{
Alberto Martorell Carreño \\ Universidad de San Martín de Porres, Perú

\section{RESUMEN}

El concepto de patrimonio mundial surge con la aprobación de la Convención del Patrimonio Mundial (CPM) de la UNESCO en 1972, documento internacional que busca promover la responsabilidad internacional hacia la conservación adecuada de bienes culturales especialmente importantes para el conjunto de la humanidad. Dado que el patrimonio es comprendido como fuente de identidades locales y nacionales, entender la relación entre los valores locales y los valores universales es un tema que atrae la atención de los especialistas. Por ello, en el contexto de la globalización, es válido preguntarse: ¿está creándose un verdadero patrimonio mundial? ¿se puede decir que la Lista del Patrimonio Mundial, es la lista del patrimonio global? ¿pertenecen los bienes inscritos en ese listado -o al menos los valores representados por dichos bienes- a la humanidad en su conjunto? ¿cuál es la posición de los sitios peruanos inscritos en el listado universal en razón a esa relación entre lo local y lo universal?

Palabras clave: Patrimonio mundial, humanidad, globalización, UNESCO.

\begin{abstract}
The concept «world heritage» arises after the approval of the World Heritage Convention of UNESCO in 1972. This document is focused in promoting the international responsibility towards the right conservation of cultural heritage goods that joint a special interest for the human community as a whole. Considering that heritage is a source for local and national identities, to understand the relationship between local and universal values is an issue that convokes the attention of the specialists. It is coherent to discuss on this relationships in the context of globalization Is it there a real process of creation of a «world heritage? Is it possible to sustain that the World Heritage List is the «global heritage list»? Do the goods inscribed (or, at least, the values represented by them) on the list represent to the human community? Which is the situation, among universal and local values, of the Peruvian sites inscribed on the universal list?
\end{abstract}

Keywords: World Heritage, humanity, globalization, UNESCO 


\section{La Lista del Patrimonio Mundial en el proceso global}

Aunque existe consenso sobre la influencia que la globalización genera en todos los ámbitos y confines de la vida, no parece haberlo con respecto a qué es la globalización (Greig, 2002). Una noción genérica nos dice que consiste en la expansión de los vínculos económicos, sociales y culturales entre los pueblos.

Hallack (1999), al referirse al rol de la UNESCO en la globalización en el campo educativo, planteaba una reforma necesaria para pasar de un modelo determinado por la lógica del mercado, la debilidad de los Estados, un concepto productivista de la educación y la violación de los derechos humanos, a uno en el que las instituciones supranacionales, inspiradas en la educación humanista universal, sean preponderantes. Sería válido aplicar las reflexiones anteriores al campo que nos ocupa.

En 1972 la UNESCO aprobó uno de los textos más importantes dentro del repertorio jurídico internacional especializado en el campo de la cultura: La Convención para la Protección del Patrimonio Mundial, Cultural y Natural (CPM). Este instrumento internacional es un referente clave para abordar el tema del patrimonio cultural y la globalización.

Pasados más de 40 años de vigencia de la CPM, son numerosos los expertos que la califican como uno de los instrumentos normativos internacionales más exitosos. No cabe duda que se trata de un texto fundamental para entender la cultura en términos globales. Los 191 Estados que forman parte de esta convención son bastante significativos. El gran reto está en pasar del concepto formal de patrimonio mundial al concepto social y global.

Mason y de la Torre (2001) se preguntan sobre si la globalización «socava (...) el significado y la eficacia de la conservación de elementos materiales». Su respuesta es provocadora, para ellos la globalización hace aún más necesario para cada cultura que ciertos aspectos de su patrimonio sean protegidos y conservados. Además, dado que la conservación es un «acto de interpretación, selección y valoración» puede contribuir a la diversidad.

La respuesta de Mason y de la Torre (2001), es afirmativa en algunas instancias:

La globalización «desterritorializa» la cultura, y por extensión, convierte a la cultura de lo material a todos los niveles en un factor de menor importancia para la vida social. Nosotros argumentamos lo contrario; es decir, los imperativos culturales para seleccionar, proteger e interpretar ciertos aspectos del mundo tangible (o en otras palabras, para conservar el patrimonio) son aún más importantes ahora para las culturas en un mundo que se globaliza.

Además, ambos autores plantean:

La conservación, lejos de dedicarse a fijar las obras culturales existentes en piedra, constituye sin lugar a dudas un medio de reinterpretar y reproducir los significados culturales en el lugar y el momento actuales. Los actos de conservación no se limitan a «mantener» bienes culturales, sus significados y sus valores (...) En segundo lugar, mantener las «reservas» de una cultura mediante la conservación representa una condición esencial para la continuidad cultural, pero también para la creatividad, que se 
sirve con frecuencia de dichas reservas (conscientemente o no). En este sentido, referirse al patrimonio físico como una forma de «capital cultural» resulta útil para conceptuar la forma en que las partes interesadas valoran, gestionan, acumulan e invierten (es decir, puede verse como un tipo de activo de capital que posee valores económicos y culturales simultáneamente). (Mason y de la Torre, 2001)

Lo anterior parece más lógico e importante cuando hablamos de bienes que, al menos en teoría, interesan a toda la humanidad.

En palabras del director general de la UNESCO, Koïchiro Matsuura (citado en Bandarin, 2005): «La Convención sobre el Patrimonio Mundial es una fuerza vital en el mundo que fomenta la coexistencia pacífica y rinde homenaje a nuestro pasado en la misma medida que a nuestro futuro».

El proceso de creación de la CPM, surgió ante la constatación de que los bienes culturales y naturales se hallaban en creciente peligro de destrucción.

Frente a ello, se afirmó que su «deterioro o (la) desaparición constituyen un empobrecimiento nefasto del patrimonio de todos los pueblos del mundo» (UNESCO, 1972) y se postuló una responsabilidad mundial por su conservación. Dicha escala supranacional se hace necesaria teniendo en cuenta que:

La protección de ese patrimonio, a escala nacional es en muchos casos incompleta, dada la magnitud de los medios que requiere y la insuficiencia de los recursos económicos, científicos y técnicos del país en cuyo territorio se encuentra el bien que ha de ser protegido. (UNESCO, 1972)

Pero esa preocupación internacional es aún anterior a la propia Convención. Resulta interesante destacar que la primera misión en el campo de la conservación de patrimonio cultural enviada por UNESCO, el año 1951, tuvo como objetivo apoyar la restauración de la ciudad del Cuzco devastada por un fuerte terremoto (Jokilehto, 2009).

Arizpe (2000) pone énfasis en el significado del patrimonio mundial para los habitantes, con un ejemplo de su propia experiencia. Relata un diálogo con un guía que demostraba especial satisfacción y orgullo cuando la acompañó en un recorrido por las iglesias barrocas de Manila (Filipinas), inscritas en la Lista del Patrimonio Mundial en 1993. Arizpe le preguntó porqué tener una placa de patrimonio mundial le resultaba importante y la respuesta fue la que sigue: Porque ahora sabemos que no son solo nuestro orgullo, sino el de toda la humanidad, y eso nos hace aún más orgullosos. La autora concluye que en esos casos el orgullo de los pocos se convierte en el orgullo de los muchos. Considera además que esa interacción entre valorización local y global otorga fuerza y continuidad a la Lista del Patrimonio Mundial.

La UNESCO (1990) declara lo siguiente:

La Convención hace viables en general, las prácticas pasadas de la Organización en cuanto a cooperación internacional para la protección del patrimonio cultural de la humanidad, al mismo tiempo que las codifica y hace extensiva dicha cooperación al patrimonio natural, cuya protección es obviamente necesaria hoy en día dado que la contaminación ambiental ha empeorado considerablemente. 
Los bienes considerados patrimonio cultural o natural protegidos por esta Convención son aquellos que tienen valor universal desde el punto de vista histórico, artístico, científico o estético.

Otra importante convención que se aboca a la protección del patrimonio cultural en la arena internacional, es la Convención para la Protección del Patrimonio Intangible - CPPI (2003). Presentan una de las dicotomías más comentadas de la globalización al reconocer:

Que los procesos de mundialización y de transformación social por un lado crean las condiciones propicias para un diálogo renovado entre las comunidades pero por el otro también traen consigo, al igual que los fenómenos de intolerancia, graves riesgos de deterioro, desaparición y destrucción del patrimonio cultural inmaterial, debido en particular a la falta de recursos para salvaguardarlo.

Sin embargo, algunos autores son muy críticos respecto al patrimonio mundial y la forma en que se ha gestionado la denominada Lista del Patrimonio Mundial. Van der Aa (2005) afirma que la actual lista es demasiado extensa y que en ella se han incluido bienes que no merecen un reconocimiento mundial. Opina este autor que la de patrimonio mundial, debería ser «la lista de lo conocido universalmente».

En todo caso, de acuerdo con las Directrices Prácticas para la Convención del Patrimonio Mundial, se trata de bienes que reúnen una característica llamada valor universal excepcional. Jokilehto destaca que, al definir la expresión valor universal excepcional, especialistas reunidos en Ámsterdam (1998) para debatir acerca de la estrategia mundial para una Lista del Patrimonio Mundial, balanceada, creíble y representativa consideraron que se trata de creaciones capaces de dar respuesta a temas de naturaleza común universal orientados hacia todas las culturas humanas u originados en todas ellas. A ello, se agregaba que en lo referente al patrimonio natural esos temas tienen que ver con la diversidad biogeográfica y que, con relación a la cultura, tienen que ver con la creatividad humana y se manifiestan en la diversidad cultural (Droste et al., 1998, citado en Jokilehto, 2009).

Gran parte de la clave está en cuál es la relación entre los valores locales y los valores universales. Bianchi y Boniface (2002) opinan que, la incorporación de monumentos y sitios que constituyen importantes repositorios de las memorias regionales en la Lista del Patrimonio Mundial, ha acentuado las tensiones acerca de los valores universales, el cosmopolitanismo, los discursos de ciudadanía y los valores simbólicos que están integrados a cada sitio.

Para Bassols (2000) el fin de la CPM fue más bien práctico. Se aprobó principalmente para «destacar la insuficiencia de la acción a nivel estatal o nacional para afrontar la protección de ciertos bienes del patrimonio cultural y natural que presentan un interés excepcional que exigen una conservación y un apoyo a nivel internacional». Es decir, que aun cuando el valor regional en todas sus expresiones tiene un rol prioritario que jugar para la comprensión de los bienes, existe una necesidad de garantizar su conservación. ¿Garantiza la CPM la conservación de los bienes inscritos bajo su virtud?

Al hablarse de valores locales y valores universales, salta a colación que se está haciendo referencia al patrimonio propio (de la población local de un punto dado) y el patrimonio del «otro» (de otra ubicación 
geográfica). Algunos autores como Lowenthal (1998) consideran que no es compatible hablar de patrimonios étnicos, locales y regionales y de patrimonio mundial.

Desde otro punto de vista, Aguilar (2001) destaca que alcanzar la conciencia suficiente para reconocer los valores de los otros, reconociendo sus diferencias, es síntoma de madurez. ¿Puede el conjunto de convenciones que la UNESCO ha promovido llevarnos a alcanzar dicha madurez a nivel global?

Francioni (2003) considera que la CPM ha sabido incorporar los avances que dentro del derecho internacional se han dado en las últimas décadas, creando un concepto en evolución, amplio y abierto. Incorpora así los principios de acción preventiva (por el que todo Estado debe tomar las medidas para prevenir los daños ambientales y, en este caso, los daños a los bienes del patrimonio cultural); el principio del patrimonio común de la humanidad (aunque el patrimonio mundial sigue estando bajo la jurisdicción nacional, se acepta cada vez más que su daño afecta a la humanidad en su conjunto), que se complementa con el principio del interés común de la humanidad; entre otros.

Merece especial atención el principio de interpretación evolutiva por el que cuando se interpreta un tratado o convención, deben utilizarse los desarrollos generales del derecho internacional, especialmente los que recaen sobre el mismo campo temático. Es importante este principio porque es el que ha permitido la evolución constante del documento aplicativo de la Convención, que son las Directrices Prácticas para la Aplicación de la Convención del Patrimonio Mundial (UNESCO, 2008), que ha sido modificado en numerosas ocasiones desde la aprobación de su primera versión, actualizando los sistemas para la evaluación, inclusión y control de los Sitios de Patrimonio Mundial (SPM) y nuevas categorías como las de paisajes e itinerarios culturales. Esos cambios se corresponden con la evolución teórica del patrimonio y del derecho internacional, pero se incorporan mediante la interpretación evolutiva al marco de la CPM, pues esta no ha sido modificada desde su aprobación original.

Los bienes que protege la CPM son aquellos que reúnen los denominados «valores universales excepcionales» que el parágrafo 49 de las Directrices Aplicativas define como: «Importancia cultural y/o natural tan excepcional que trasciende las fronteras y es de común importancia para las presentes y futuras generaciones de la humanidad en su conjunto» (UNESCO, 2008). De allí que se declare el interés de la comunidad internacional por su conservación.

Por supuesto, esa definición sigue siendo bastante genérica y solo se puede entender aplicándola caso a caso, a la luz de los denominados criterios (parágrafo 77 de las directrices aplicativas). Para ello, los organismos evaluadores (ICOMOS y la UICN) emiten una opinión técnica que es evaluada por el organismo encargado de la gestión del Comité del Patrimonio Mundial.

La aplicación de la CPM en los primeros lustros, fue materia de críticas que dieron lugar a la estrategia global para una Lista del Patrimonio Mundial, más balanceada, creíble y representativa.

En opinión de ICOMOS (2004), organismo asesor de UNESCO especializado en la CPM, se desprende que las deficiencias existentes en la Lista del Patrimonio Mundial, responden a dos tipos de razones: estructurales y cualitativas: 
Las limitaciones estructurales se relacionan con la falta de capacidad técnica para promover y preparar nominaciones, llevar adelante evaluaciones adecuadas de los bienes patrimoniales o la falta de un marco legal o de gestión adecuado. Esos factores, ya sea independientemente o sumados, dificultan la preparaciones de nominaciones exitosas...

Cualitativas: el segundo grupo de limitaciones se refieren a los vacíos en la Lista del Patrimonio Mundial, los cuales pueden verse como asociados a ciertos tipos de bienes o temas. Una manera de enfrentar esos vacíos es promover la preparación de Listas Indicativas de los Estados Partes que no cuentan con ellas. Sin embargo, para que estas Listas reflejen la totalidad de bienes culturales de los países de manera adecuada, deben considerarse nuevas vías para la identificación de bienes culturales. Se sugiere que una vía positiva en este sentido podría ser la elaboración de Listas Indicativas basadas en la evaluación de los valores universales excepcionales de los bienes potenciales, reflejando la evaluación que se haría cuando sus candidaturas para patrimonio mundial sean presentadas.

UNESCO (1990) explica como...

Al adoptar la Estrategia Global, el Comité del Patrimonio Mundial deseaba ampliar la definición del patrimonio mundial para reflejar mejor todo el espectro de nuestros tesoros culturales y naturales mundiales y proveer un marco comprehensivo y una metodología operativa para implementar la Convención del Patrimonio Mundial.

Esta nueva visión va más allá de las definiciones estrechas de patrimonio y se esfuerza por reconocer y proteger los sitios que son demostraciones sobresalientes de la coexistencia humana en el territorio así como las interacciones humanas, la coexistencia cultural, la espiritualidad y la expresión creativa.

La Estrategia Global se plantea que:

Para asegurar el porvenir de una Lista del Patrimonio Mundial más representativa, equilibrada y creíble, se necesita la presencia de bienes culturales y naturales de tipos, regiones y épocas que están menos representados. De igual manera, resulta indispensable considerar las nuevas concepciones sobre el patrimonio cultural que se han visto enriquecidas desde hace dos décadas. (CONACULTA-INAH, 2003).

Surge para superar los siguientes desbalances:

- Desbalance entre sitios culturales y naturales. Siendo mayor el número de bienes culturales que el de naturales.

- Desbalance entre los bienes terrestres y marinos. De acuerdo a los análisis del Programa de las Naciones Unidas para el Medio Ambiente (PNUMA) y del Centro para el Monitoreo de la Conservación Mundial, los sitios marinos y costeros tienen poca presencia en la Lista.

- Desbalance entre la atención dada a la inscripción del sitio y la gestión del sitio. Se trata de una problemática sumamente seria. El proceso de inscripción de bienes en la Lista durante los primeros años 
de su funcionamiento, no tuvo el nivel de exigencia en cuanto se refiere a las garantías administrativas, a las que agregaríamos las jurídicas y políticas, que demuestren en primer lugar que el Estado Parte que presentaba el bien estuviese realmente dispuesto a tomar medidas para su real conservación. Un ejemplo de lo anterior es el del Santuario Histórico-Natural de Machu Picchu (inscrito en 1983). Al momento de inscribirlo en 1983 no se presentó un Plan de Gestión, el mismo que solo formalmente se aprobó en 1998, pero como respuesta a intereses políticos coyunturales y no a la creación de las condiciones de sostenibilidad que el sitio requiere. La situación que a inicios del año 2010 se está viviendo, responde a una evidente falta de planificación y de capacidad de gestión.

- Desbalance entre inscripciones de sitios simples e inscripciones seriadas. Las inscripciones seriadas implican dos o más sitios que no tienen una conexión física entre sí, pero que sin embargo guardan relación por provenir del mismo grupo histórico-cultural; por tratarse de un mismo tipo de bien característico de una zona geográfica; o pertenecer o la misma formación geológica o geomorfológica, la misma provincia biogeográfica o el mismo tipo de ecosistema (Directrices Prácticas, parágrafo 137). Los casos de esta naturaleza son menores a los de bienes individuales.

Esos desbalances son consecuencias de una sobrerepresentación de Europa con relación a otras regiones en la Lista del Patrimonio Mundial. Además a que el número de sitios históricos, monumentales y vinculados con la religión católica es también mucho mayor que al de bienes provenientes de otros contextos, entre otros factores.

La Estrategia Global responde inicialmente a cuatro objetivos, conocidos como las «cuatro c»:

- Fortalecer la credibilidad de la Lista del Patrimonio Mundial, como testimonio representativo y teográficamente balanceado de los bienes culturales y naturales de valor universal;

- Asegurar la conservación efectiva de los bienes del patrimonio mundial;

- Promover el desarrollo de medidas para el fortalecimiento de capacidades (capacity building);

- Incrementar la conciencia pública, el sentimiento de relación y el apoyo a la conservación mediante la comunicación.

Una «quinta $\mathrm{c}$ » fue agregada al conjunto anterior durante la $31^{\circ}$ Sesión del Comité del Patrimonio Mundial, el año 2007. Se trata de la participación de las comunidades locales. Fue promovido por Nueva Zelanda, país anfitrión de aquella sesión. Entonces los Estados Parte de la CPM declararon que la identificación, gestión y conservación del patrimonio deben hacerse, cuando sea posible, involucrando activa y significativamente a las comunidades humanas, superando los problemas de exclusión y omisión de las comunidades locales.

En Sevilla (2009) se presentaron ante el Comité del Patrimonio Mundial los resultados de la aplicación de la Estrategia hasta el momento, considerándolos bastante positivos en algunos aspectos. Se destaca, por ejemplo, en cuanto a la representatividad que el $79 \%$ de los Estados Parte tienen ya bienes inscritos en la Lista. Ello significa que durante el periodo 2003-2009, 22 Estados Parte habían inscrito por primera vez un bien en la Lista del Patrimonio Mundial. La representatividad también sería mayor porque del total de 890 bienes inscritos 
al momento de la reunión comentada, 116, equivalentes al 13\% estaban ubicados en las regiones subrepresentadas (África, el Caribe y el Pacífico). Esto se debe a que el 19\% de las inscripciones nuevas corresponden a dichas regiones.

Un aspecto que se considera más difícil de evaluar es el del equilibrio. Se destaca sobre ese punto que el objetivo es «pasar de una visión puramente arquitectónica del patrimonio cultural de la humanidad a una visión mucho más antropológica, multifuncional y universal». Se relaciona este punto con el de los paisajes culturales, llegando a 36 los inscritos entre 2003 y 2009, un 22\% del número total de bienes inscritos en ese periodo. Es de esperarse que la progresiva importancia que deben ir ganando los itinerarios culturales contribuya seriamente a una lista balanceada.

\section{Patrimonio mundial en el caso peruano}

Luego de la revisión de la literatura existente realizada en los párrafos precedentes, dedicaremos las líneas sucesivas a la participación del Perú como país miembro del CPM.

Debemos decir que, salvo por la involucración que nuestro país ha tenido en el proceso de gestación y avances del llamado Programa Qhapaq Ñan, nacido por una iniciativa del gobierno de Valentín Pañiagua y posteriormente convertido en un proyecto internacional que involucra a Argentina, Bolivia, Chile, Colombia, Ecuador y al propio Perú con el apoyo técnico del Centro del Patrimonio Mundial y la participación financiera de otros organismos como el BID, la participación del Perú en la Lista del Patrimonio Mundial resulta pobre en cuanto a representatividad, con relación a la importancia de los procesos históricos que se han vivido en nuestro territorio nacional, y con relación a la capacidad de gestión de los sitios ya inscritos. El Programa Qhapaq Ñan y sus contrapartes en los países mencionados y la UNESCO han logrado que el año 2014 este bien sea inscrito como patrimonio seriado.

A la fecha, hay 8 sitios peruanos incluidos en la Lista del Patrimonio Mundial como bienes culturales.

1. Ciudad histórica del Cusco (1983).

2. Sitio arqueológico de Chavín (1985).

3. Zona arqueológica de Chan Chan (que fue inscrita a su vez en la Lista del Patrimonio Mundial y en la Lista del Patrimonio Mundial en Peligro, 1986).

4. Centro Histórico de Lima (1988).

5. Líneas y Geoglifos de Nazca y Pampas de Jumana (1994).

6. Centro Histórico de Arequipa (2000).

7. Ciudad Sagrada de Caral-Supe (2009).

Hay que agregar a lo anterior un sitio seriado más, también correspondiente a los bienes cutlurales: 
8. El Qhapaq Ñan, Camino Principal Andino (2014).

Además, se han inscrito como bienes naturales dos sitios:

9. Parque Nacional de Huascarán (1985).

10. Parque Nacional del Manu (1987) .

Dos más ocupan la categoría de bienes mixtos (aquellos que presentan «independientemente» tanto valores culturales como naturales universales y excepcionales):

11. Santuario histórico de Machu Picchu (1983).

12. Parque Nacional de Río Abiseo (1988).

Si pensamos, como hemos comentado, en que el Perú es uno de los territorios donde se han gestado procesos civilizatorios de la mayor importancia mundial, la participación peruana en la Lista del Patrimonio Mundial resulta bastante limitada.

Para dar una idea comparada podemos citar el caso de México, país que ha basado gran parte de su estrategia de desarrollo turístico en la capacidad de convocatoria de la Lista del Patrimonio Mundial para el turismo internacional. Cuenta con un total de 32 sitios inscritos, habiendo presentado a razón de una candidatura por año entre los años 2006 y 2014. Si comparamos ese mismo periodo, el Perú no formalizó ninguna candidatura. Lo hizo sí, el año 2009, con Caral, sitio inscrito luego de 9 años del anterior (el Centro Histórico de Arequipa) y luego de 5 más, con el Qhapaq Ñan.

No pretendemos afirmar que el hecho de inscribir bienes en la Lista del Patrimonio Mundial deba convertirse en un objetivo de la política cultural en sí misma. Pero sí consideramos que es sistemático que no se hayan realizado esfuerzos por mejorar la participación peruana, dada la positiva repercusión que en la imagen internacional de los países tiene este hecho, y los compromisos hacia la conservación y uso sostenible de los bienes que implica.

De acuerdo a los criterios de la Estrategia Mundial, el Perú no es un país subrepresentado. Sin embargo, habría muchas posibilidades a considerar a partir de la lógica de la propia estrategia. Es sintomático que ninguno de los bienes peruanos incluidos en la Lista Indicativa sean «paisajes culturales» cuando todas las reuniones internacionales en las que se ha debatido el tema presentan como el más importante paisaje cultural sudamericano el Cañón del Colca. Dice mucho también que sitios tan notables como el de Sipán, las Huacas del Sol y de la Luna, el Complejo Arqueológico del Brujo, las Pirámides de Túcume, entre otros, no se encuentren en el referido listado.

Como se ha mencionado, la gestión de la CPM está a cargo de un organismo denominado el Comité del Patrimonio Mundial, integrado por los representantes de los Estados elegidos por la Asamblea General y del que el Perú ha formado parte en tres ocasiones: entre 1989 y 1995, y entre 2004 y 2009; y, actualmente, 
desde el 2014. Cabe preguntarse cuáles han sido los aportes de nuestro país a la gestión del patrimonio mundial. Resulta extraño entonces, salvo por explicaciones políticas, que hayamos sido elegidos nuevamente para esta honrosa posición.

No se trata de una crítica únicamente a las instituciones públicas. Igual pregunta cabría formular con respecto a la participación en los organismos internacionales especializados. Es especialmente influyente el ICOMOS. El Perú no ha tenido un representante en el Comité Ejecutivo de dicha organización desde hace más de 15 años. El liderazgo en la subregión lo ejerce Argentina, que sí ha participado constantemente en las reuniones internacionales con aportes concretos y ha recibido encargos internacionales.

¿No sería lógica una participación mucho más activa de la comunidad científica nacional especializada cuando nos encontramos en el país que tiene la mayor riqueza monumental del subcontinente?

El mecanismo establecido para el proceso de inscripción de bienes en la Lista del Patrimonio Mundial, implica un paso anterior, formal y mandatorio, que es manifestar ante la UNESCO la intención del Estado Parte de presentar la candidatura de determinado bien, inscribiéndola en su respectiva Lista Indicativa. Mientras que México tiene 24 sitios en su Lista Indicativa nacional, el Perú tiene 7.

Por otro lado, el estado de conservación y la capacidad de gestión del Estado peruano con relación a los sitios ya inscritos en la Lista del Patrimonio Mundial siguen dejando mucho que desear. Veremos a continuación los principales casos:

- Chan Chan. Tantos años en la Lista del Patrimonio Mundial como en la Lista del Patrimonio Mundial en Peligro. Es cierto que por sus características, al ser la ciudadela de barro más extensa del mundo, su conservación es especialmente frágil. No deja de ser preocupante, pese a lo anterior, que un bien pueda mantenerse en situación de peligro por más de 2 décadas. Si bien se sabe que hay una serie de esfuerzos locales por la conservación, incluyendo la creación de una Unidad Ejecutora en los últimos años, también resulta necesario tener en cuenta que el principal riesgo existente en el sitio, que es el nivel de la capa freática y los niveles de humedad que ello genera en detrimento de las estructuras de tierra, parece haberse alterado negativamente debido a la irrigación de Chavimochic. Algunas labores de han venido realizando para la recuperación o encausamiento de las aguas subterráneas que la irrigación genera. Debe hacerse un gran esfuerzo en ese sentido.

- Santuario Histórico de Machu Picchu. Se trata de un caso sumamente complejo y solo podemos referirnos muy referencialmente a él. Como dijimos, la carencia total de un sistema de planificación hasta el año de 1998, y la aprobación de un Plan de Gestión inspirado en intereses políticos del momento, hacen que Machu Picchu se encuentre en una situación realmente preocupante.

El crecimiento caótico e incontrolado de Aguas Calientes, que en una jugada política pasó a reconocerse oficialmente como «Machu Picchu Pueblo» con el nivel de una municipalidad distrital, es un caso interesante para que reflexionemos sobre los resultados del hecho mismo de inscribir un bien como patrimonio mundial. ¿Son necesariamente positivos? ¿Generan beneficios para las «poblaciones locales? ¿Son fuente de desarrollo? 
Las inundaciones que a inicios del 2010 han tenido lugar, nuevamente, en la zona de Machu Picchu, deberían hacernos reflexionar sobre lo que viene sucediendo. La sobreexposición mediática del bien a raíz de su inscripción como «maravilla del mundo» parece haber potenciado aún más su fama e incrementado la presión del turismo mundial por visitarlo. Los hechos parecen estar demostrando que el desarrollo turístico implica mucho más que la existencia del atractivo e inclusive de cierta infraestructura para atender a los visitantes. Implica una política sostenible y coherente que, por ejemplo, evite que un bien se convierta en atractivo único y sin el cual ninguna alternativa interesante puede ser ofrecida a los visitantes.

Aguas Calientes es presentado por sus pobladores actuales como un caso de desarrollo económico espontáneo que beneficia a sectores desfavorecidos de la población local. Lo que podría tomarse como verdad, resulta incluso falso si uno analiza los hechos desde una perspectiva más amplia. Hay algunos factores a considerar: primero, la mayor parte de la población que dio lugar al centro poblado de Aguas Calientes no puede ser calificada como población local. Se trataba originalmente de una población flotante que atendía los servicios básicos de los visitantes que el tren dejaba en la estación del lugar. Era contado el número de establecimientos como pequeños restaurantes y hoteles, básicamente orientados a atender a los viajeros que llegaban a través del llamado Camino Inca.

La verdadera población local de Machu Picchu la constituyen las dispersas comunidades campesinas autóctonas, que prácticamente no son mencionadas en el Plan Maestro del año 1998 y han sido sistemáticamente ignoradas en la mayor parte de los documentos oficiales. La versión actualizada del Plan mediante Resolución Directoral 738-INC de 8 de junio de 2005, cuya plena ejecución aún no se ha implementado, contiene un ejemplo de desarrollo que vincula al turismo con la comunidad local, en la zona de Piscaicucho, punto de inicio del Camino Inca, donde la comunidad administra un local de parqueo de buses, baños y venta de artesanía y alimentos para el recorrido.

¿Hasta qué punto los pobladores locales se han visto, entonces, beneficiados con la inscripción de Machu Picchu en la Lista del Patrimonio Mundial? ¿Hasta qué punto la población local ha comprendido la relación de derechos y deberes que vivir en la región donde se halla uno de los bienes más emblemáticos del mundo implica?

Si tenemos en cuanta la magnitud de las pérdidas económicas que se vienen sucediendo, a raíz de suspensiones del uso turístico por sucesos naturales como el del 2010, que expertos del Observatorio Turístico del Perú han calculado en 400 millones de dólares, cabe preguntarnos nuevamente si la presencia de un bien «único» en el que se basa aparentemente toda la lógica del crecimiento del turismo receptivo de manera espontánea, benéfica o no a la población local, es fuente o no de desarrollo sostenible y si, por otro lado, las condiciones para la adecuada conservación del bien están siendo verdaderamente respetadas.

En la versión revisada del Plan Maestro de Machu Picchu se planteó una visión regional del sistema de gestión, considerando los potenciales logros positivos que debería generar el Plan de Reordenamiento y Rehabilitación del Valle del Vilcanota que se viene llevando adelante por convenio con el Banco Mundial y el gobierno del Perú desde el año 2005. Pero resulta absolutamente censurable que en tantos años de elaboración de ese Plan Maestro, hasta el día de hoy no esté aprobado. 
El Plan Maestro dice al respecto: Los diversos estudios realizados afirman que Machu Picchu no se explica de forma aislada, sino en el conjunto del Valle Sagrado, y por tanto, alcanzaría su impacto y protección a diversas ciudades y pueblos incas: Pisac, Yucay y Ollantaytambo, etc. que, inclusive rebasaría la actual área del Santuario Histórico. Existe la necesidad de dar un tratamiento de protección integral al conjunto de la cuenca del río Vilcanota en Urubamba, garantizando su desarrollo sostenible.

Sin lugar a dudas es necesario partir de una visión ecosistémica, pero también de una visión de la integridad cultural del área en cuestión.

- $\quad$ El Centro Histórico de Arequipa. Un tercer caso que queremos citar es el de la ciudad de Arequipa, cuya inscripción en la Lista del Patrimonio Mundial data del año 2000. Numerosas tensiones se han presentado debido a proyectos de desarrollo, siendo el más discutido en los últimos años la construcción de un puente que atravesaría la zona paisajística de la ciudad, en el sector de Chilina. UNESCO ha considerado que esto dañaría los valores universales excepcionales de la ciudad histórica. A la fecha, sin embargo, el puente está culminado y en funcionamiento, sin que parezca haberse generado nuevas preocupaciones de primer nivel para la UNESCO.

- El Centro Histórico de Lima. Queremos terminar este breve recuento del estado de la conservación de bienes culturales inscritos en la Lista del Patrimonio Mundial en el Perú, con el preocupante caso de Lima al 2010. Desde que la nueva gestión asumiera la gestión de Lima, se han dado algunos anuncios sumamente procupantes, siendo el más grave el de una posible demolición de cerca a un $40 \%$ de sus inmuebles por hallarse en mal estado de conservación. Esto es inaceptable. Un proceso de gestión adecuado puede llevar a la recuperación de una gran parte de los inmuebles incluidos en ese porcentaje, y un sistema de protección que dinamice mecanismos de inversión a través de procesos controlados de renovación urbana, de métodos de incentivo económico adecuados, de gestión pública-privada, entre otros, llevaría a una recuperación integral, en la que no se lleve de paso a soluciones fáciles como la anunciada demolición. Por otra parte, hay otros temas que preocupan, como la falta de respuesta del Estado peruano y la Municipalidad Metropolitana de Lima de manera específica, a las observaciones que UNESCO ha hecho a las instalaciones del corredor segmentado, «Metropolitano», a su paso por el centro histórico. No podemos dejar de manifestar que el problema es integral y no de determinado grupo responsable de la conducción política de Lima. El hecho de que en todo un periodo no se haya logrado la aprobación de un nuevo Plan Maestro del Centro Histórico de Lima es altamente significativo. 


\section{Conclusiones}

Formulamos dos conclusiones finales, la primera relativa al tema del patrimonio mundial en el proceso de globalización, y la segunda referida al caso peruano:

1. El rol de la Lista del Patrimonio Mundial en el proceso de globalización es sumamente importante, pues puede efectivamente contribuir a la configuración de un proceso más sostenible, de encuentro de valores entre los pueblos, generando un nuevo simbolismo de carácter universal que contribuya al desarrollo de relaciones interculturales y la generación de paz.

2. La situación de los sitios peruanos inscritos en la LPM es sumamente preocupante. El Estado peruano debería asumir una política cultural más responsable hacia estos bienes, no solo por los deberes asumidos ante la colectividad internacional, sino y sobre todo por la importancia de dichos bienes para el ser nacional y la identidad peruana. 


\section{Referencias}

Arizpe, L. (2000). Cultural Heritage and Globalization. En Values and Heritage Conservation. Research report. The Getty Conservation Institute, Los Ángeles.

Bandarin, F. (s/f). La Convención sobre el Patrimonio Mundial: 30 años después. Recuperado de http:/www.juntadeandalucia.es/ cultura/iaph/publicaciones/dossiers/dossier13/dossier13art1.htm

Bassols, M. (2000). Conjuntos históricos y rehabilitación urbana: recomendaciones internacionales y normativa autonómica, especial consideración de la Ley de la Comunidad de Madrid sobre rehabilitación de espacios urbanos degastados y de los inmuebles a preservar. En Asamblea. Revista parlamentaria de la Asamblea de Madrid, 21-52.

Bianchi, R. \& Boniface, P. (2002). Editorial: the politics of World Heritage. International Journal of Heritage Studies, 8(2), 79-80.

CONACULTA-INAH (2003). La Representatividad en la Lista del Patrimonio Mundial. El Patrimonio Cultural y Natural de Iberoamérica, Canadá y Estados Unidos. México DF.

Francioni, F. (2003). Treinta años después: ¿está la Convención sobre el Patrimonio Mundial preparada para el siglo XXI? Patrimonio Cultural y Derecho, 7, 11-38.

Greig, J. M. (2002). The End of Geography?: Globalization, Communications, and Culture in the International System. The Journal of Conflict Resolution, 46(2), 225-243.

Hallack, J. (1999). Globalization, human rights and education. International Institute for Educational Planning (IIEP/UNESCO) working paper, 33. Paris: UNESCO.

ICOMOS, (2004). The World Heritage List Filling the Gaps - an Action Plan for the Future. ICOMOS, Paris.

Jokilehto, J. (2009). ICCROM and the Conservation of Cultural Heritage. A history of the Organization's first 50 years, $1959-2009$. Roma: ICCROM.

Lowenthal, D. (1998). The heritage crusade and the spoils of history. Cambridge: University Press.

Mason, R. \& de la Torre, M. (2001). Heritage Conservation and Values in Globalizing Societies. Recuperado de http:// www.minervapartners.org/html/consvalues.pdf.

UNESCO (1990). Convenciones y recomendaciones de la UNESCO sobre la protección del patrimonio cultural. Lima: PNUD/ UNESCO. Recuperado de http://whc.unesco.org/en/globalstrategy

Van der Aa, B. J. M. (2005). Preserving the heritage of humanity? Obtaining world heritage status and the impacts of listing (Tesis doctoral). University of Groningen. 